\title{
BTN3A2 Expression Is Connected With Favorable Prognosis and High Infiltrating Immune in Lung Adenocarcinoma
}

\section{Hao Zhou}

The Affiliated Suzhou Science\&Technology Town Hospital of Nanjing Medical University Yuansheng Lin ( $\sim$ linys202012@163.com)

The Affiliated Suzhou Science\&Technology Town Hospital of Nanjing Medical University

\section{Research Article}

Keywords: BTN3A2, prognosis, immune infiltration, LUAD

Posted Date: June 14th, 2021

DOl: https://doi.org/10.21203/rs.3.rs-562967/v1

License: (c) (1) This work is licensed under a Creative Commons Attribution 4.0 International License.

Read Full License 


\section{Abstract}

Background: butyrophilin subfamily 3 member A2 (BTN3A2) as a important mediator in immune activation, its reported to be linked to many cancers progress. However, the relation with infiltrating immune and prognosis of BTN3A2 in Lung adenocarcinoma are not clear.

Methods: In our study, we checked the mRNA expression and protein expression profile of BTN3A2 in Lung adenocarcinoma (LUAD) and its relation to clinical outcomes using TIMER, UALCAN database. Besides, we analyzed the survival of BTN3A2 in LUAD by Kaplan-Meier Plotter and PrognoScan database. Moreover, we analyzed Gene Set Enrichment Analysis (GSEA) of the BTN3A2. Next, we explored the relation of BTN3A2 expression with the immune infiltration by TIMER. At last, In order to enrich the regulatory mechanism of BTN3A2, we used miRarbase, starbase and miRDB dadabase to look for miRNAs targets of BTN3A2.

Results: the mRNA along with protein expression of BTN3A2 in the LUAD group was lower in contrast with that in the normal group. Besides, high BTN3A2 expression was connected with good first progression (FP) and overall survival (OS) in LUAD. Then, the GSEA analysis demonstrated that T cell receptor signaling cascade, B cell receptor signaling cascade, natural killer cell mediated cytotoxicity, immune receptor activity, immunological synapse, T cell activation were enriched differentially in BTN3A2 high expression phenotype of LUAD. Moreover, BTN3A2 expression is remarkable positively correlation with invading levels of tumor purity, B cells, neutrophil, CD4+ T cells, dendritic cells, macrophages, and CD8+ T cells in LUAD, and B cells and dendritic cells were linked with good prognosis of LUAD. To further enrich the possible regulatory mechanisms of BTN3A2, we analyzed the miRNA targets. The results showed that hsa-miR-17-5p may be miRNA targets of BTN3A2.

Conclusion: Taking together, we provide evidence of BTN3A2 as possible prognosis biomarkers of LUAD. Besides, high BTN3A2 expression in LUAD may influence prognosis because of immune invasion. Moreover, our findings provide a potential mechanism that hsa-miR-17-5p may be miRNA targets of BTN3A2.

\section{Background}

Lung cancer is the most frequent cause of cancer-linked deaths universal. 1.8 million people are diagnosed with lung cancer and 1.6 million people die of the lung cancer each year[1]. In spite of advances in therapy choices, such as irradiation, iatrochemistry, surgery and targeted treatment, prognosis remains poor due to the presence metastatic cancers in most patients at the moment of diagnosis[2, 3]. Thus, finding appropriate prognostic biomarker is crucial for LUAD patients.

Butyrophilin (BTN) family members are immunoglobulin like molecules, which act as immune checkpoint modulators and play a role in self tolerance. BTN3 family members include BTN3A1, BTN3A2 and BTN3A3. The expression of BTN3A2 in neonatal autoimmune and allergic diseases has a strong causal relationship[4]. BTN3A2 expression is regulated by stress, which is linked to the prognosis of pancreatic 
cancer, and may constitute an immune escape mechanism to prevent $\mathrm{V}_{Y} 9 \mathrm{~V} \delta 2 \mathrm{~T}$ cell recognition [5]. Studies suggest that BTN3A2 is differentially expressed in a variety of cancers. BTN3A2 is expressed in triple-negative breast cancer, pancreatic ductal carcinoma, and epithelial ovarian cancer, and is associated with good prognosis $[6,7]$. BTN3A2 is highly expressed and repressed the growth, migration and infiltration of gastric cancer cells $[8,9]$. Nevertheless, the role of BTN3A2 in LUAD is unclear.

Herein, we first explored the mRNA along with protein expression of BTN3A2 in the LUAD TIMER, UALCAN database. Then, we used Kaplan-Meier Plotter database to analyze the survival of BTN3A2 in LUAD. Next, we utilized UALCAN database and GSEA software to analyze the BTN3A2 promoter methylation level. Moreover, To explore the relationship of BTN3A2 expression with the immune infiltration, we used TIMER database. At last, we analyzed miRNA targets to enrich the possible regulatory mechanism of BTN3A2. The results showed that high BTN3A2 expression in LUAD may influence prognosis because of immune invasion. Moreover, our findings provide a potential mechanism that hsa-miR-17-5p may be miRNA targets of BTN3A2.

\section{Methods}

\section{UALCAN analysis}

We analyzed the protein expression along with mRNA expression levels of SKA3 in UALCAN data resource (http://ualcan.path.uab.edu/index.html)[10].

\section{TIMER analysis}

BTN3A2 expression in cancers was determined in the TIMER data resource (https://cistrome.shinyapps.io/timer/)[11]. We identified the expression of BTN3A2 in different tumors by TIMER data resource. After that, the association of BTN3A2 with immune invasion in LUAD was estimated by TIMER algorithm. Furthermore, the relationship of ACE2 with the type biomarkers of tumor purity, $B$ cells, CD $8+T$ cells, CD $4+T$ cells, macrophages, neutrophil and dendritic cells in LUAD were verified.

Human Protein Atlas

We used Human Protein Atlas (https://www.proteinatlas.org/) to detect BTN3A2 protein expression in immunohistochemistry.

\section{Kaplan-Meier plotter databases}

We employed the Kaplan-Meier plotter data resource (http://kmplot.com/)[12] to explore the value of BTN3A2 expression in LUAD prognosis. This threshold was logrank $p$ value $<0.05$ in Kaplan-Meier plotter data resource.

\section{PrognoScan database}


We analyzed the prognosis in LUAD by PrognoScan database

(http://dna00.bio.kyutech.ac.jp/PrognoScan/index.html)[13]. Cox P-value < 0.05 Considered significant difference.

\section{GSEA analysis}

GSEA was performed to clarify the molecular mechanisms of the prognostic gene signature. GSEA was performed in GSEA v. 3.0 were searched to determine the enriched biological processes, cellular components, molecular functions, KEGG pathway associated with survival of the high-risk group. FDR < 0.05 and $|\mathrm{NES}|>1$ were considered statistically significant.

To further explore the targets of BTN3A2 in LUAD, we used miRarbase, starbase and miRDB dadabase to predicted miRNA-target genes of BTN3A2. Moreover, the target site in the BTN3A2 3'UTR were predicted to pair with hsa-miR-17-5p by miRDB (http://www.mirdb.org/)[14]and RNA22Sites (https://cm.jefferson.edu/rna22v2.0/)[15].

\section{Statistical analysis}

The results of the survival analysis were acquired from a log-rank test. We used spearman's correlation to evaluate BTN3A2 with immune invasion and type biomarkers of immune cells. comparing two independent samples was using Student's t test. $P<0.05$ signified statistical significance.

\section{Results}

\section{BTN3A2 expression levels in different human cancers and LUAD.}

The study design is shown as a flowchart for the analysis of the BTN3A2 gene (Fig. 1A). To detect BTN3A2 expression in cancer and non-malignant tissues, the BTN3A2 mRNA contents in different cancer type tissues were analyzed using the sangerbox and TIMER data resources. The results illustrated that the BTN3A2 expression was lower in LUAD, Lung squamous cell carcinoma, as well as Kidney renal clear cell carcinoma in contrast with the non-malignant tissues (Fig. 1B). Moreover, data from TCGA demonstrated that the BTN3A2 content was lower in the LUAD group in contrast with the healthy group (Fig. 1C-1D). Interestingly, protein expression of BTN3A2 in LUAD was still much lower in contrast with that in non-malignant tissues from CPTAC samples (UALCAN) (Fig. 1E). To verify the histological level of BTN3A2, we used the Human Protein Atlas database. The results showed that BTN3A2 is upregulated in normal tissue and downregulated in LUAD tissues (Fig. 7F).

\section{BTN3A2 transcription in subgroups of patients with LUAD, stratified based on gender, race, nodal status metastasis and other criteria (UALCAN).}

To further analysis multiple clinic features of 515 LUAD samples in the TCGA exhibited low BTN3A2 mRNA content. BTN3A2 mRNA content was lower in LUAD patients in contrast with healthy individuals in subgroup analyses on the basis of gender, race, nodal metastasis, smoking, stages, as well as tumor grade (Fig. 2). Therefore, BTN3A2 levels may have promising LUAD diagnostic value. 


\section{Prognostic Potential of BTN3A2 in LUAD}

To explore whether BTN3A2 expression was related to prognosis of LUAD patients, we evaluated the influence of BTN3A2 contents on OS and FP through the Kaplan-Meier plotter data resource. The data demonstrated the high BTN3A2 contents were linked to good prognosis in LUAD from 204820_s_at probe (OS HR $=0.71, \mathrm{P}=8.5 \mathrm{e}-08 ; \mathrm{FP} \mathrm{HR}=0.71, \mathrm{P}=5.5 \mathrm{e}-04)$ (Fig. 3A-3B). The results were consistent with these in 209846_s_at probe and 212613_s_at probe from Kaplan-Meier plotter (Fig. 3C-3F). To further verify the Prognostic value of BTN3A2 in LUAD, PrognoScan database was used and the results showed that the low BTN3A2 expression group was linked to good lung cancer prognosis (OS GSE31210 HR $=1.55, P=$ 0.034; RFS GSE31210 HR = 1.26, $P=0.0093)(F i g .3 G-3 H)$. Thus, our data suggest that low BTN3A2 content is a protective factor and results in good prognosis in LUAD.

\section{GSEA showed the most significant pathways and genes related with BTN3A2 based on TCGA}

To identify the different signal pathways activated in LUAD, we used GSEA between high and low BTN3A2 expression data sets. 6 hallmark gene-sets (including

KEGG_T_CELL_RECEPTOR_SIGNALING_CASCADE, KEGG_B_CELL_RECEPTOR_SIGNALING_CASCADE, KEGG_NATURAL_KILLER_CELL_MEDIATED_CYTOTOXICITY, GO_IMMUNE_RECEPTOR_ACTIVITY, GO_IMMUNOLOGICAL_SYNAPSE, GO_T_CELL_ACTIVATION) were chosen for analysis (Fig. 4A-4F). The results showed that $T$ cell receptor signaling cascade, $B$ cell receptor signaling cascade, natural killer cell mediated cytotoxicity, immune receptor activity, immunological synapse, $\mathrm{T}$ cell activation are differentially enriched in BTN3A2 high expression phenotype of LUAD.

\section{Relationship between expression of BTN3A2 and immune invasion in LUAD}

To assess the potential relationship of immune invasion with BTN3A2 expression in LUAD, we used TIMER to conduct the following analysis. Firstly, the "Gene" module analysis revealed that BTN3A2 expression is remarkable positively correlation with invading levels of tumor purity, B cells, dendritic cells, CD8 + T cells, macrophages, neutrophil and CD4 + T cells in LUAD (Figure. 5A). Then, the "SCNA" module analysis revealed immune cell infiltration may relevant to altered BTN3A2 gene copy numbers, including $B$ cells, dendritic cells, CD $4+T$ cells, macrophages, $C D 8+T$ cells, and neutrophils in LUAD (Figure. 5B). Besides, the "SURVIVAL" module analysis demonstrated that high $\mathrm{B}$ cell and dendritic cell levels were linked to good prognosis of LUAD (Figure. $5 C, P<0.05$, respectively).

To study the relationship between immune cell markers and BTN3A2 expression, we analyzed the markers of $B$ cells, macrophages, CD $8+T$ cells, dendritic cells and neutrophils by TIMER data resource. Interestingly, we discovered that the expression levels of CD19 along with CD79A in B cells, CD8B and CD8A in CD8 + T cell, CEACAM8, ITGAM and CCR7 in neutrophils, T helper cells (Th1 and Th2),

Macrophage, Dendritic cell have markedly positive related to BTN3A2 expression in LUAD (table).

The data imply that high BTN3A2 expression in LUAD may influence prognosis because of immune invasion.

\section{6. hsa-miR-17-5p may be miRNA targets of BTN3A2}


To further elucidate the miRNA targets of BTN3A2 in LUAD, we used miRarbase, starbase and miRDB dadabase to predicted miRNA-target genes of BTN3A2 showing in the venn plot (Fig. 6A). The result showed that it had 6 miRNA-target (hsa-miR-17-5p; hsa-miR-20a-5p; hsa-miR-93-5p; hsa-miR-106b-5p; hsa-miR-20b-5p; hsa-miR-519d-3p) of BTN3A2 and which was visualized in Fig. 6B. Then, we analyzed prognostic potential of 6 miRNA-target in lung cancer by Kaplan-Meier Plotter database. Interestingly, we found that only hsa-miR-17 had differential expression $(P=1.62 E-12)$ and prognostic value $(O S, H R=1.4$, $P=0.035)$ (Fig. 6C-6D). Moreover, the target site in the BTN3A2 3'UTR were predicted to pair with hsa-miR17-5p by miRDB and RNA22Sites (Fig. 6E).

We have confirmed that BTN3A2 expression was relation to the immune infiltration in LUAD, and the height expression of BTN3A2 was also correlated with the better prognosis of LUAD. Therefore, we hypothesized that hsa-miR-17 expression that may be the miRNA targets of BTN3A2. Prognosis analysis on the basis of the expression of hsa-miR-17of LUAD in linked immune cells subtype was performed using Kaplan Meier plotter, and data illustrated that the high hsa-miR-17 content of LUAD in abundant B cells $(H R=0.03)$, abundant $C D 4+$ memory $T$ cells $(H R=0.042)$, abundant macrophages $(H R=0.04)$, abundant Regulatory T cells $(H R=0.031)$ and Type $2 \mathrm{~T}$ helper cells $(H R=0.0077)$ had poor prognosis respectively (Fig. 7A-7E). Thus, the results may provide a potential mechanism that hsa-miR-17-5p may be miRNA targets of BTN3A2.

\section{Discussion}

Lung cancer is the primary cause of cancer death due to its poor prognosis and high aggressiveness. Immunotherapy, especially the emergence of immune checkpoint inhibitors (ICIS), has changed the therapeutic prospect of non-small cell lung cancer[16-18]. The development of lung cancer is a complex process involving interactions between stromal fibroblasts, immune cells, and tumor cells. Tumor infiltrating immune cells have an important role in inhibiting or promoting tumor growth $[19,20]$. The immune cell response is related to the clinical prognosis of Lung cancer, and cancer linked memory $T$ cells can anti-tumor immunity of Lung cancer [21]. Hence, it is essential to illustrate the relationship between lung cancer and immune invasion, and identify an immune linked biomarker for the prognosis of lung cancer.

BTN3A2 is highly expressed in gastric cancer (GC) tissues, and the deletion of BTN3A2 can inhibit the proliferation, invasion and migration of GC cells [22]. Studies showed that the abundance of $\gamma \delta T$ cells was positively correlated with the expression of BTN3A1/BTN3A2/BTN3A3, suggesting that BTN family proteins may be involved in the activation and proliferation of $\gamma \delta T$ cells in the HNSCC tumor microenvironment[23]. BTN3A2 gene has Just a few studies, and it is related to tumor target, prognosis and immunity.

GSEA is the most commonly used statistical methods[24]. Considering the fact that the prognosis of BTN3A2 in LUAD is unclear, we firstly explored BTN3A2-related GSEA. GSEA results indicated that T cell receptor signaling cascade, $B$ cell receptor signaling cascade, natural killer cell mediated cytotoxicity, 
immune receptor activity, immunological synapse, T cell activation are differentially enriched in BTN3A2 high expression of LUAD. Multiple pathways were activated and these pathways are associated with immune infiltration.

BTN3A2 is associated with prognosis of breast cancer (BRCA) and ovarian cancer (OV), and immunoinfiltration in triple-negative breast cancer (TNBC) and OV are highly associated with BTN3A2[24]. Studies have shown that prognosis of LUAD is linked to immune invasion [27-29]. Herein, Our study is consistent with previous studies and results show that BTN3A2 expression was remarkable positively linked to invading levels of tumor purity, B cells, neutrophils, CD8 + T cells, macrophages, dendritic cells, and CD $4+T$ cells, in LUAD, and B cells and dendritic cells were linked with good prognosis of LUADBTN3A2. The results indicated that the results suggested that BTN3A2 expression is connected with favorable prognosis in LUAD and high BTN3A2 expression in LUAD may affect prognoses due to immune infiltration.

BTN3A is a prognostic marker and target of $\mathrm{V} Y \mathrm{~V} \delta 2 \mathrm{~T}$ cell immunotherapy for pancreatic ductal adenocarcinoma (PDAC)[30]. To further illustrate whether BTN3A2 had other regulated mechanisms in LUAD. Multiple website tools were used to confirm BTN3A2-target miRNAs, hsa-miR-17-5p was obtained. The result showed hsa-miR-17-5p was over-expressed in LUAD tissues, which might be another mechanisms for down-regulated expression of BTN3A2. hsa-miR-17-5p was differentially expressed in numerous cancer types and its over-expression could indicate a poorer survival in lung cancer. In the meantime, hsa-miR-17 was also Confirmed to cause EMT process through regulating CYP7B1 expression in colon cancer[32]. These aberrant regulation of hsa-miR-17 would help us to know the prognosis of BTN3A2.

Prognosis analysis on the basis of the expression of hsa-miR-17of LUAD in linked immune cells subtype was performed using the Kaplan Meier plotter. The data demonstrated that the high expression of hsamiR-17 of LUAD in abundant B cells, CD $4+$ memory $T$ cells, macrophages, Regulatory $T$ cells and Type 2 $T$ helper cells had poor prognosis respectively (Fig. 7A-7E). Thus, the results may provide a potential mechanism that hat hsa-miR-17-5p may be miRNA targets of BTN3A2 in LUAD.

\section{Conclusions}

In summary, we provide evidence of BTN3A2 as possible prognosis biomarkers of LUAD. Besides, high BTN3A2 expression in LUAD may influence prognosis because of immune invasion. Moreover, our findings provide a potential mechanism that hsa-miR-17-5p may be miRNA targets of BTN3A2 in LUAD.

\section{Abbreviations}

LUAD: Lung adenocarcinoma; PFS: progression-free survival; BTN3A2: butyrophilin subfamily 3 member A2; OS: overall survival; Cl: Confidence Intervals; HR: Hazard Ratio; TCGA: The Cancer Genome Atlas. 


\section{Declarations}

\section{Ethics approval and consent to participate}

Not applicable.

\section{Consent for publication}

Not applicable.

\section{Availability of data and materials}

We downloaded RNA-Seq gene expression profiles, and clinical data for LUAD types from the TCGA data portal (https://portal.gdc.cancer.gov/). The data included 535 tumor samples and 59 normal samples.

\section{Competing interests}

The authors declare that they have no competing interests.

\section{Funding}

No funding was obtained for this study.

\section{Author Contributions}

YSL conceived the project. $\mathrm{HZ}$ analyzed the data. YSL and $\mathrm{HZ}$ contributed towards the interpretation of the data. All authors contributed to the article and approved the final manuscript.

\section{Acknowledgements}

Not applicable.

\section{References}

1. Hirsch, F.R., et al., Lung cancer: current therapies and new targeted treatments. Lancet, 2017. 389(10066): p. 299-311.

2. Altorki, N.K., et al., The lung microenvironment: an important regulator of tumour growth and metastasis. Nat Rev Cancer, 2019. 19(1): p. 9-31.

3. Chen, Z., et al., Non-small-cell lung cancers: a heterogeneous set of diseases. Nat Rev Cancer, 2014. 14(8): p. 535-46.

4. Huang, Q.Q., et al., Neonatal genetics of gene expression reveal potential origins of autoimmune and allergic disease risk. Nat Commun, 2020. 11(1): p. 3761.

5. Vantourout, P., et al., Heteromeric interactions regulate butyrophilin (BTN) and BTN-like molecules governing $\gamma \delta$ T cell biology. Proc Natl Acad Sci U S A, 2018. 115(5): p. 1039-1044. 
6. Cai, P., et al., BTN3A2 serves as a prognostic marker and favors immune infiltration in triple-negative breast cancer. J Cell Biochem, 2020. 121(3): p. 2643-2654.

7. Le Page, C., et al., BTN3A2 expression in epithelial ovarian cancer is associated with higher tumor infiltrating T cells and a better prognosis. PLoS One, 2012. 7(6): p. e38541.

8. Zhu, M., et al., Exome Array Analysis Identifies Variants in SPOCD1 and BTN3A2 That Affect Risk for Gastric Cancer. Gastroenterology, 2017. 152(8): p. 2011-2021.

9. Han, J., et al., MMP11 and CD2 as novel prognostic factors in hormone receptor-negative, HER2positive breast cancer. Breast Cancer Res Treat, 2017. 164(1): p. 41-56.

10. Chandrashekar, D.S., et al., UALCAN: A Portal for Facilitating Tumor Subgroup Gene Expression and Survival Analyses. Neoplasia (New York, N.Y.), 2017. 19(8): p. 649-658.

11. Li, T., et al., TIMER: A Web Server for Comprehensive Analysis of Tumor-Infiltrating Immune Cells. Cancer Res, 2017. 77(21): p. e108-e110.

12. Györffy, B., et al., An online survival analysis tool to rapidly assess the effect of 22,277 genes on breast cancer prognosis using microarray data of 1,809 patients. Breast Cancer Res Treat, 2010. 123(3): p. 725-31.

13. Mizuno, $\mathrm{H}$., et al., PrognoScan: a new database for meta-analysis of the prognostic value of genes. BMC Med Genomics, 2009. 2: p. 18.

14. Chen, Y. and X. Wang, miRDB: an online database for prediction of functional microRNA targets. Nucleic Acids Res, 2020. 48(D1): p. D127-D131.

15. Miranda, K.C., et al., A pattern-based method for the identification of MicroRNA binding sites and their corresponding heteroduplexes. Cell, 2006. 126(6): p. 1203-17.

16. Sun, S., J.H. Schiller and A.F. Gazdar, Lung cancer in never smokers-a different disease. Nat Rev Cancer, 2007. 7(10): p. 778-90.

17. Peng, S., et al., EGFR-TKI resistance promotes immune escape in lung cancer via increased PD-L1 expression. Mol Cancer, 2019. 18(1): p. 165.

18. Rosner, S., J.E. Reuss and P.M. Forde, PD-1 Blockade in Early-Stage Lung Cancer. Annu Rev Med, 2019. 70: p. 425-435.

19. Wang, S.S., et al., Tumor-infiltrating B cells: their role and application in anti-tumor immunity in lung cancer. Cell Mol Immunol, 2019. 16(1): p. 6-18.

20. Öjlert, Å.K., et al., The immune microenvironment in non-small cell lung cancer is predictive of prognosis after surgery. Mol Oncol, 2019. 13(5): p. 1166-1179.

21. Snyder, M.E. and D.L. Farber, Human lung tissue resident memory $T$ cells in health and disease. Curr Opin Immunol, 2019. 59: p. 101-108.

22. Zhu, M., et al., Exome Array Analysis Identifies Variants in SPOCD1 and BTN3A2 That Affect Risk for Gastric Cancer. Gastroenterology, 2017. 152(8): p. 2011-2021.

23. Lu, H., et al., High Abundance of Intratumoral y $\mathrm{T}$ Cells Favors a Better Prognosis in Head and Neck Squamous Cell Carcinoma: A Bioinformatic Analysis. Front Immunol, 2020. 11: p. 573920. 
24. Subramanian, A., et al., Gene set enrichment analysis: a knowledge-based approach for interpreting genome-wide expression profiles. Proc Natl Acad Sci U S A, 2005. 102(43): p. 15545-50.

25. Cai, $P$., et al., BTN3A2 serves as a prognostic marker and favors immune infiltration in triple-negative breast cancer. J Cell Biochem, 2020. 121(3): p. 2643-2654.

26. Le Page, C., et al., BTN3A2 expression in epithelial ovarian cancer is associated with higher tumor infiltrating T cells and a better prognosis. PLoS One, 2012. 7(6): p. e38541.

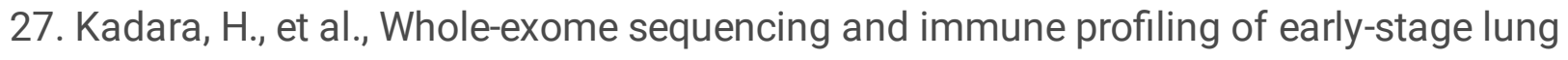
adenocarcinoma with fully annotated clinical follow-up. Ann Oncol, 2017. 28(1): p. 75-82.

28. Ghorani, E., et al., Differential binding affinity of mutated peptides for MHC class I is a predictor of survival in advanced lung cancer and melanoma. Ann Oncol, 2018. 29(1): p. 271-279.

29. Gong, Z., et al., Impaired Cytolytic Activity and Loss of Clonal Neoantigens in Elderly Patients With Lung Adenocarcinoma. J Thorac Oncol, 2019. 14(5): p. 857-866.

30. Benyamine, A., et al., BTN3A is a prognosis marker and a promising target for $\mathrm{V} Y \mathrm{~V}$ V $2 \mathrm{~T}$ cells basedimmunotherapy in pancreatic ductal adenocarcinoma (PDAC). Oncoimmunology, 2017. 7(1): p. e1372080.

31. Chen, Q., et al., Prognostic significance of serum miR-17-5p in lung cancer. Med Oncol, 2013. 30(1): p. 353.

32. Xi, X.P., et al., MicroRNA-17 induces epithelial-mesenchymal transition consistent with the cancer stem cell phenotype by regulating CYP7B1 expression in colon cancer. Int J Mol Med, 2016. 38(2): p. 499-506.

\section{Table}


Table 1

Correlation analysis between BTN3A2 and immune cell type markers in TIMER database.

\section{Description Gene markers}

\begin{tabular}{|c|c|c|c|c|c|c|c|}
\hline & & \multicolumn{2}{|l|}{ None } & \multicolumn{2}{|l|}{ Tumor } & \multicolumn{2}{|l|}{ Age } \\
\hline & & $\mathrm{COR}$ & $P$ & COR & $P$ & COR & $P$ \\
\hline \multirow[t]{2}{*}{$\mathrm{CD} 8+\mathrm{T}$ cell } & CD8A & 0.534 & $<0.001$ & 0.448 & $9.30 \mathrm{e}-26$ & 0.542 & $3.71 e-38$ \\
\hline & CD8B & 0.416 & $<0.001$ & 0.329 & $6.62 \mathrm{e}-14$ & 0.425 & $1.30 \mathrm{e}-22$ \\
\hline \multirow[t]{2}{*}{$\mathrm{T}$ cell } & CD3E & 0.62 & $4.79 e-56$ & 0.552 & $1.27 e-40$ & 0.624 & $1.48 e-53$ \\
\hline & CD2 & 0.601 & $7.67 e-52$ & 0.526 & $2.24 \mathrm{e}-36$ & 0.604 & $2.44 \mathrm{e}-49$ \\
\hline \multirow[t]{2}{*}{ B cell } & CD19 & 0.373 & $1.96 \mathrm{e}-18$ & 0.246 & $3.32 \mathrm{e}-08$ & 0.393 & $2.98 \mathrm{e}-19$ \\
\hline & CD79A & 0.354 & $1.07 e-16$ & 0.23 & $2.46 \mathrm{e}-07$ & 0.372 & $2.61 e-17$ \\
\hline \multirow[t]{3}{*}{ Macrophage } & INOS (NOS2) & 0.091 & $3.99 \mathrm{e}-2$ & 0.006 & $8.86 \mathrm{e}-01$ & 0.093 & $4.04 \mathrm{e}-02$ \\
\hline & IRF5 & 0.371 & $<0.001$ & 0.298 & $1.36 \mathrm{e}-11$ & 0.38 & $4.38 \mathrm{e}-18$ \\
\hline & COX2(PTGS2) & 0.089 & $4.27 e-02$ & -0.099 & $2.84 \mathrm{e}-02$ & -0.086 & $5.88 \mathrm{e}-02$ \\
\hline \multirow[t]{4}{*}{ Dendritic cell } & HLA-DQB1 & 0.437 & $1.95 e-25$ & 0.363 & $8.45 e-17$ & 0.437 & $6.30 \mathrm{e}-24$ \\
\hline & HLA-DRA & 0.556 & $<0.001$ & 0.49 & $3.40 e-31$ & 0.546 & $6.23 e-39$ \\
\hline & HLA-DPA1 & 0.597 & $<0.001$ & 0.544 & $2.96 \mathrm{e}-39$ & 0.587 & $5.14 \mathrm{e}-46$ \\
\hline & BDCA-1(CD1C) & 0.288 & $2.75 e-11$ & 0.226 & $3.82 \mathrm{e}-07$ & 0.267 & $2.66 \mathrm{e}-09$ \\
\hline \multirow[t]{3}{*}{ Neutrophils } & CD66b(CEACAM8) & 0.114 & $9.6 \mathrm{e}-03$ & 0.11 & $1.44 \mathrm{e}-02$ & 0.085 & $6.34 \mathrm{e}-02$ \\
\hline & CD11b (ITGAM) & 0.485 & $<0.001$ & 0.418 & $2.86 e-22$ & 0.486 & $5.44 \mathrm{e}-30$ \\
\hline & CCR7 & 0.516 & $<0.001$ & 0.436 & $3.10 e-24$ & 0.508 & $5.52 \mathrm{e}-33$ \\
\hline \multirow[t]{5}{*}{ Th1 } & TBX21 & 0.565 & $<0.001$ & 0.5 & $1.52 \mathrm{e}-32$ & 0.572 & $2.57 e-43$ \\
\hline & STAT4 & 0.519 & $7.53 e-37$ & 0.44 & $8.39 e-35$ & 0.522 & $3.46 \mathrm{e}-35$ \\
\hline & STAT1 & 0.554 & $<0.001$ & 0.496 & $5.25 e-32$ & 0.567 & $1.75 e-42$ \\
\hline & IFN-y(IFNG) & 0.431 & $1.03 e-24$ & 0.354 & $5.23 e-16$ & 0.445 & $8.05 e-25$ \\
\hline & TNF-a (TNF) & 0.312 & $6.02 e-13$ & 0.204 & $5.10 \mathrm{e}-06$ & 0.312 & $2.33 e-12$ \\
\hline \multirow[t]{2}{*}{ Th2 } & GATA3 & 0.552 & $2.01 e-42$ & 0.477 & $1.93 e-29$ & 0.556 & $1.68 \mathrm{e}-40$ \\
\hline & STAT6 & 0.181 & $3.72 \mathrm{e}-05$ & 0.209 & $2.93 e-06$ & 0.171 & $1.54 \mathrm{e}-04$ \\
\hline
\end{tabular}

LUAD, Lung adenocarcinoma; COR, R value of Spearman's correlation 


\begin{tabular}{|clllllll|}
\hline Description & Gene markers & COAD & & & & \\
\hline & STAT5A & 0.564 & $1.20 \mathrm{e}-44$ & 0.497 & $4.12 \mathrm{e}-32$ & 0.569 & $8.13 \mathrm{e}-43$ \\
\hline IL13 & 0.166 & $1.57 \mathrm{e}-04$ & 0.106 & $1.87 \mathrm{e}-02$ & 0.165 & $2.60 \mathrm{e}-04$ \\
\hline
\end{tabular}

Figures 
Figure 1
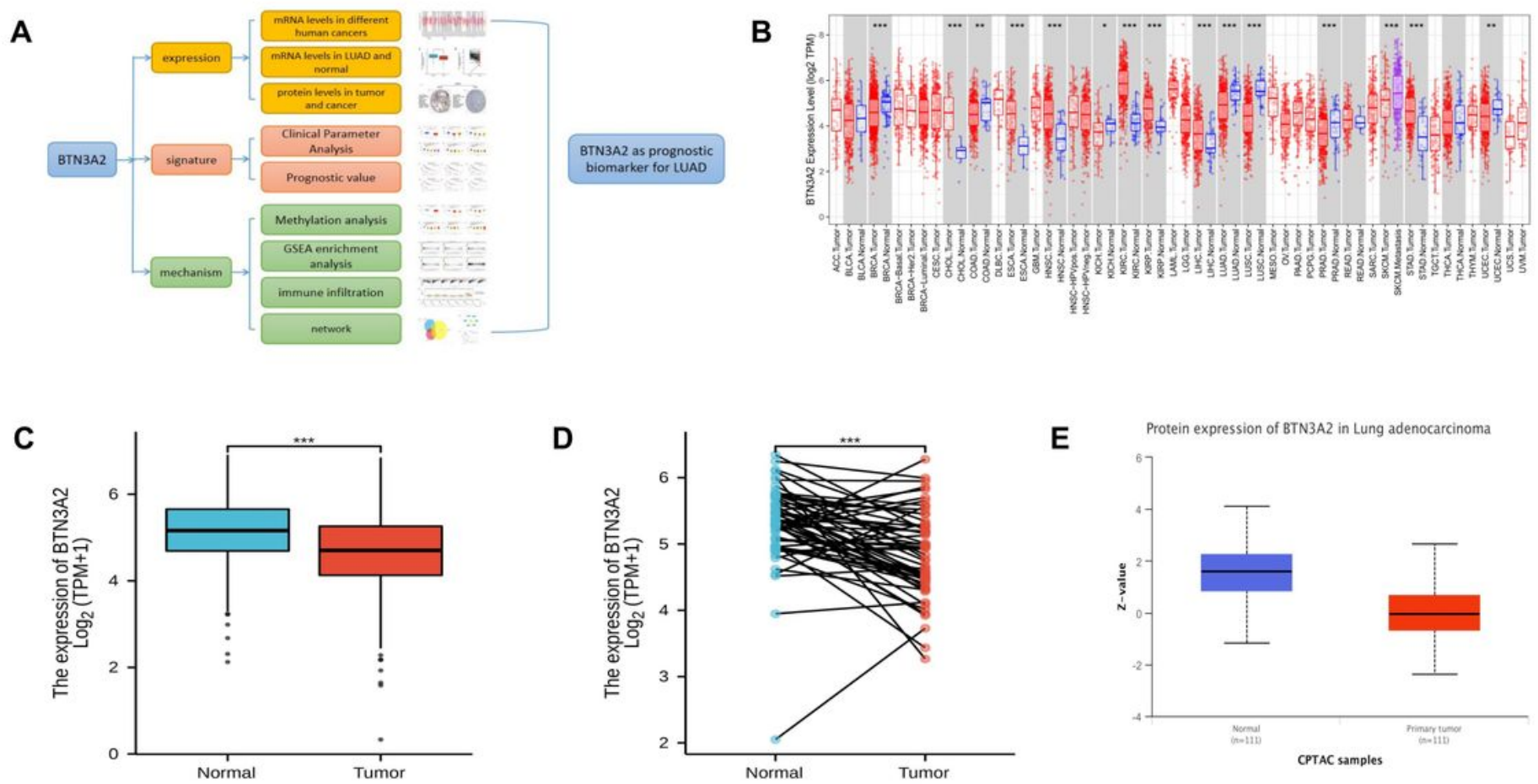

F

BTN3A2

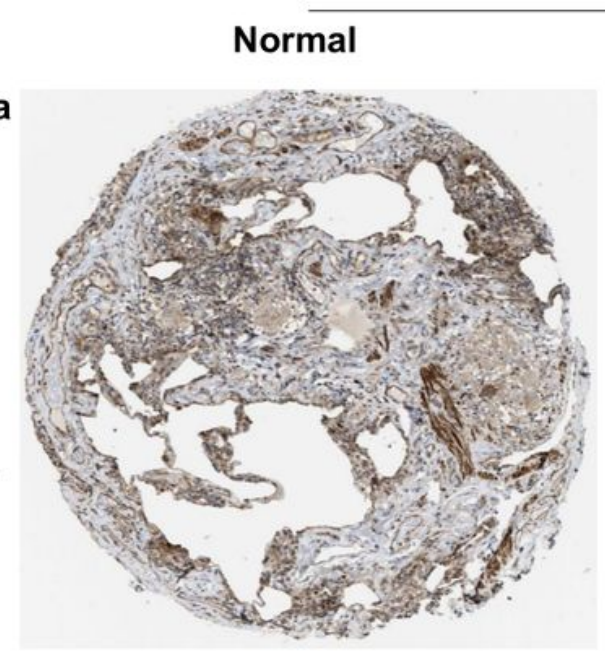

Lung (T-28000)

Adenocarcinoma, NOS

(M-81403)

Patient id: 3003

CAB019392

Male, age 49

Staining:Not detected

Intensity:Negative

Quantity:None b

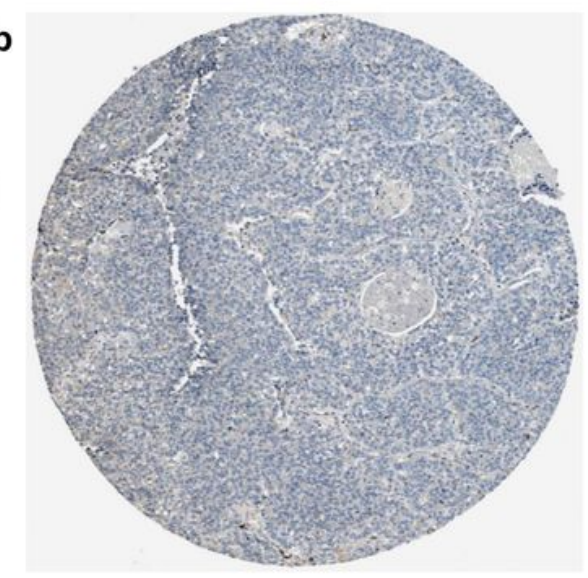

\section{Figure 1}

BTN3A2 contents in various human cancers and LUAD. (A). Workflow of this study. (B). BTN3A2 contents in different cancer from TCGA data resource were analyzed by TIMER. (C). BTN3A2 contents in nonmalignant and tumor tissues of LUAD patients in TCGA database. (D). BTN3A2 contents in pre-disease and post-disease form the same sample. (E). Ualcan database showed the protein expression of BTN3A2 in non-malignant and LUAD groups from CPTAC samples. (F). Expression of BTN3A2 in LUAD samples and normal tissues in the Human Protein Atlas. 
Figure 2
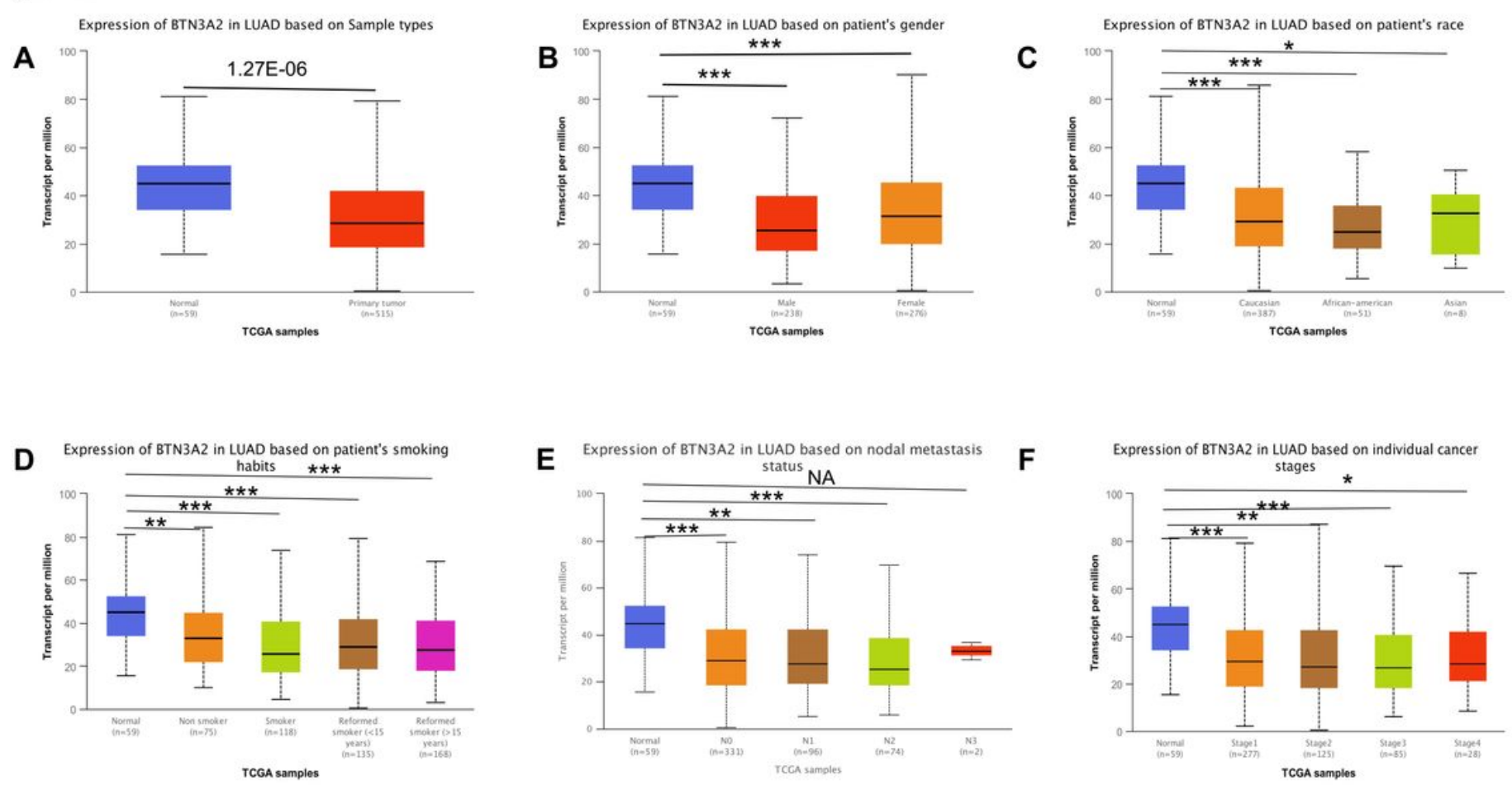

Figure 2

BTN3A2 contents in LUAD (UALCAN). (A-F). BTN3A2 contents were low in LUAD. (NA, $P>0.05, *, P<0.05$, $* *, P<0.01, * * *, P<0.001)$.

Figure 3
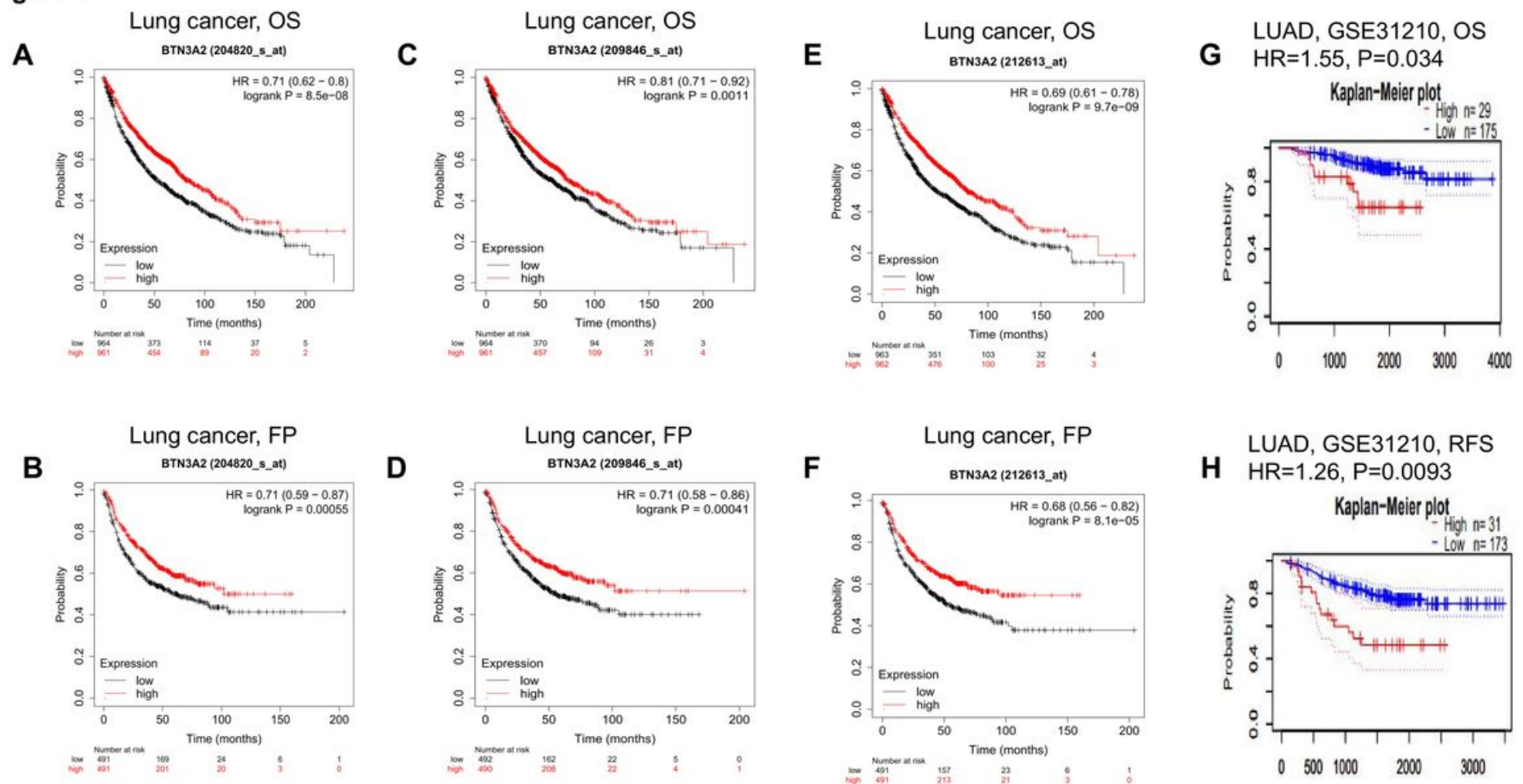

LUAD, GSE31210, RFS

H $H R=1.26, P=0.0093$

Kaplan-Meier plot

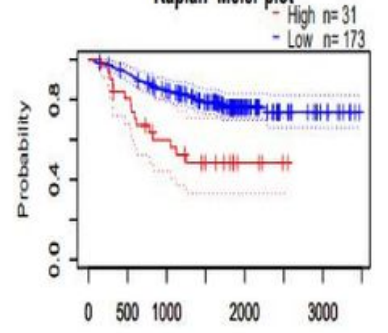




\section{Figure 3}

Comparing the high and low expression of BTN3A2 in LUAD by Kaplan-Meier survival curves. (A-B). OS and FP survival curves of lung cancer in 204820_s_at probe $(n=1295, n=982)$. (C-D). OS and FP survival curves of lung cancer in 209846_s_at probe. $(n=1295, n=982)$. (E-F). FP and OS survival curves of lung cancer in 212613_s_at probe $(\mathrm{n}=1295, \mathrm{n}=982)$. $(\mathrm{G}-\mathrm{H})$. OS and RFS survival curves of LUAD in GSE31210. OS: overall survival; FP: First progression; RFS: Relapse Free Survival.

\section{Figure 4}

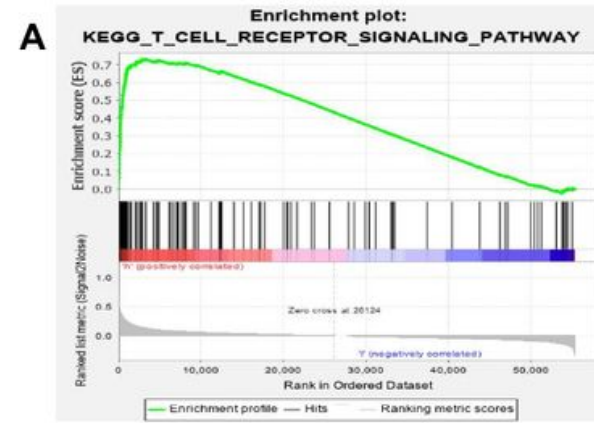

D

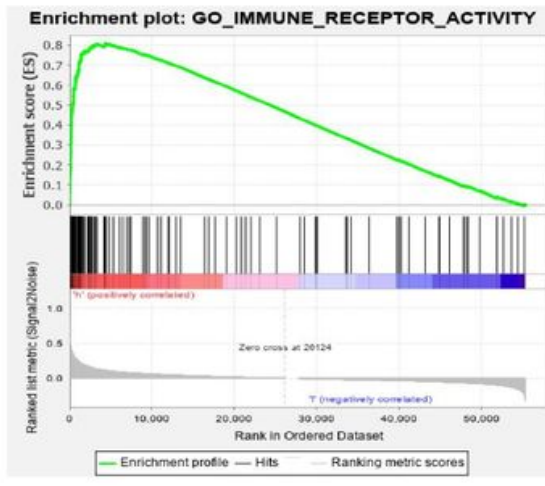

B
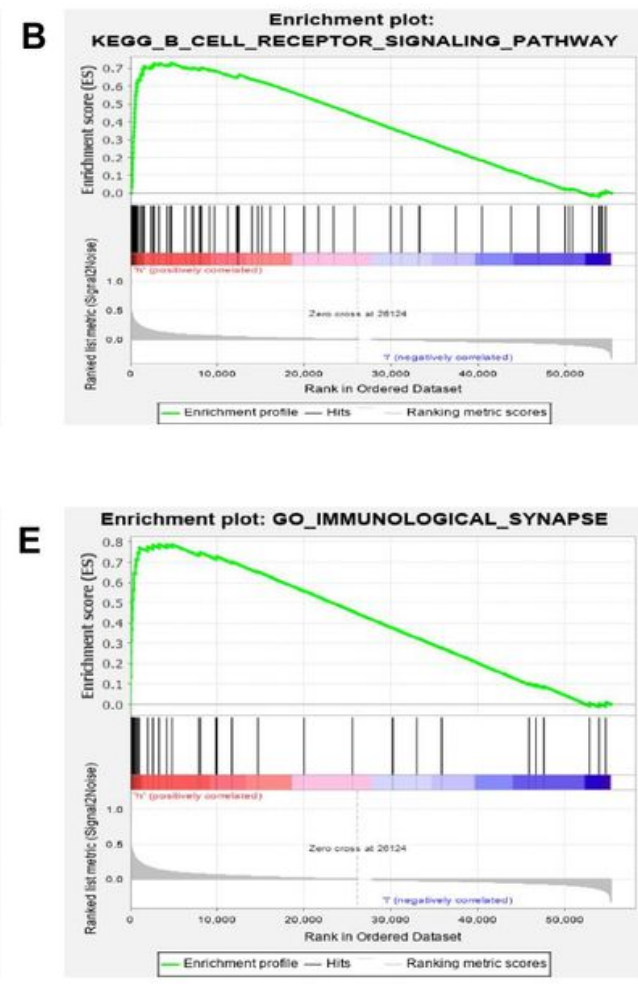

C Kegg_natural KiLrichment plot:
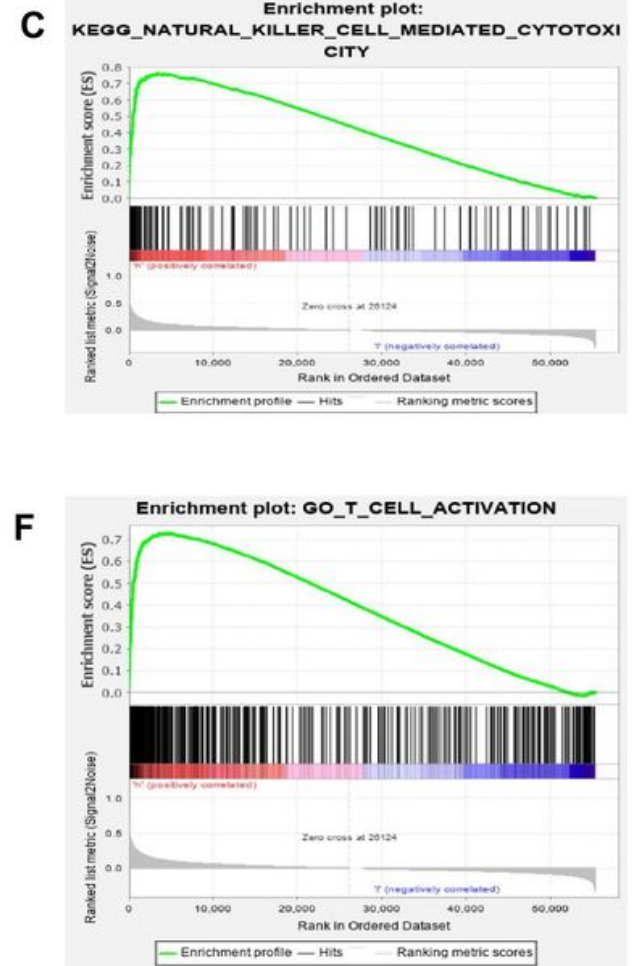

Figure 4

Gene set enrichment analysis (GSEA) indicated the most significant pathways and genes related with BTN3A2 based on TCGA. GSEA results showing gene sets in 6 hallmark gene-sets, including (A)

“KEGG_T_CELL_RECEPTOR_SIGNALING_CASCADE”, (B)

"KEGG_B_CELL_RECEPTOR_SIGNALING_CASCADE”, (C)

"KEGG_NATURAL_KILLER_CELL_MEDIATED_CYTOTOXICITY”, (D) "GO_IMMUNE_RECEPTOR_ACTIVITY”, (E) "GO_IMMUNOLOGICAL_SYNAPSE", (F) “GO_T_CELL_ACTIVATION”. 
Figure 5

A 홍

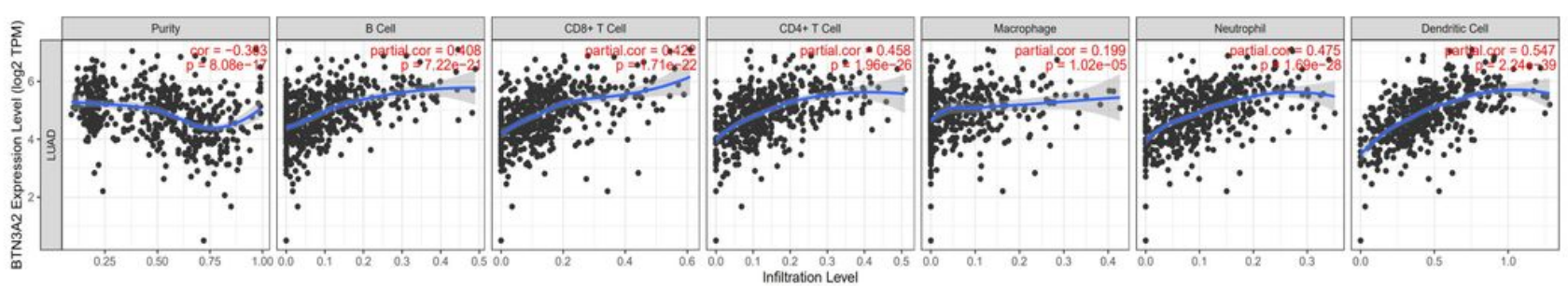

B

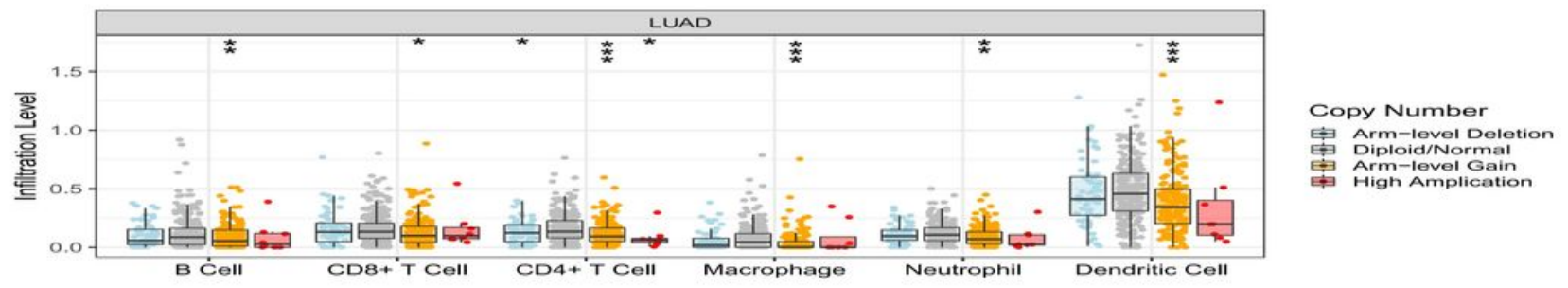

C



Figure 5

The expression of BTN3A2 in LUAD was linked to immune invasion. (A). BTN3A2 expression has positively correlations with the infiltration levels of purity, B cells, neutrophils, CD8+ T cells, dendritic cells, CD4+ T cells, and macrophages in LUAD. (B). BTN3A2 CNV influences the infiltration level of B cells, CD8+ T cells, CD4+ T cells, macrophages, neutrophil and dendritic cell in LUAD. (C). Kaplan-Meier plots of the relationship of immune invasion with OS of LUAD. ${ }^{*} p<0.05$; ${ }^{*} p<0.01 ;{ }^{*}{ }^{*} p<0.001$. 
Figure 6

A

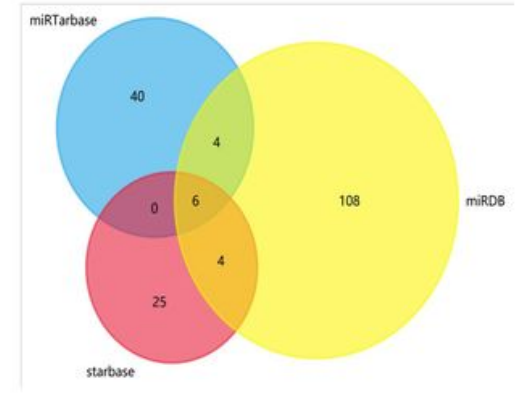

D

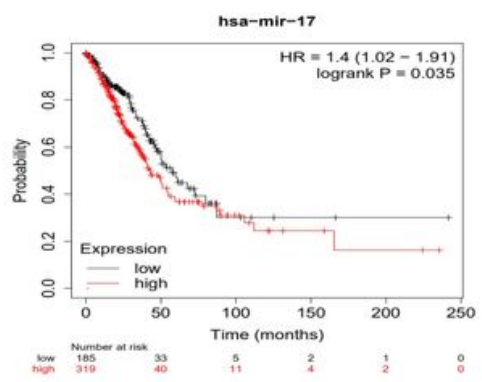

B



C

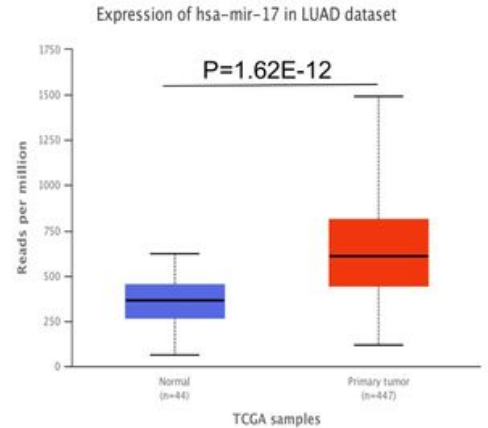

E

BTN3A2 3'UTR $\quad$ 5'- ATGCCTGTAATCCTAGCACTTTG-3'

hsa_miR_17_5p 3'-GATGGACGTGACATTCGTGAAAC-5'

\section{Figure 6}

hsa-miR-17-5p may be the target of BTN3A2. (A). The Venn shows miRNA-target genes predicted by miRarbase, starbase and miRDB. (B). miRNA-target regulatory network (Cytoscape). (C). Expression of hsa-miR-17 in UALCAN database. (D). Survival curve of hsa-miR-17 in lung cancer by Kaplan-Meier Plotter database. (E). Base pairing between hsa_miR_17_5p and the target site in the BTN3A2 3'UTR predicted by miRDB and RNA22Sites. 
Figure 7

LUAD, B cells, enriched

A

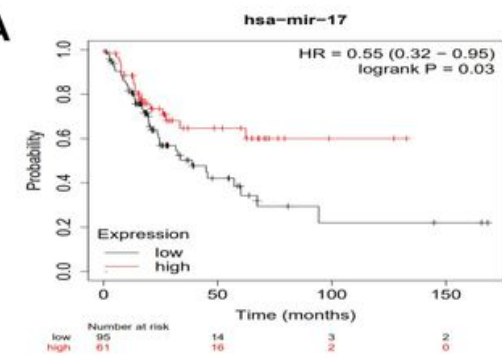

LUAD, Regulatory T cells, enriched

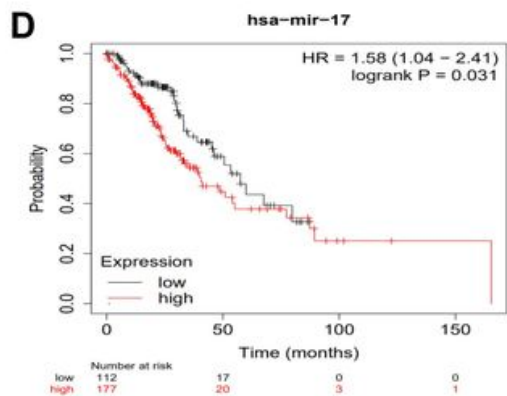

LUAD, CD4+ memory T cells, enriched

B

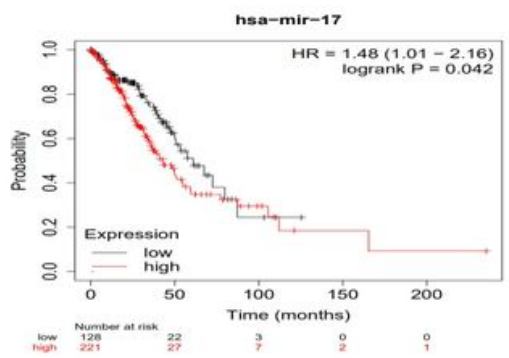

LUAD, Type 2 T helper cells, enriched

E

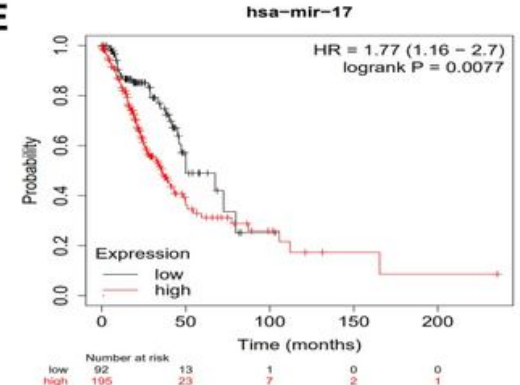

LUAD, Macrophages, enriched



\section{Figure 7}

Correlations between hsa-miR-17 expression and immune infiltration in LUAD. Kaplan-Meier survival curves compared between over-expression and down-expression of hsa-miR-17 in LUAD on the basis of immune cells subtypes. Association between LUAD of different immune cells subtype and prognosis (AE). 